\title{
An Eternal Challenge: Assessment and Documentation of Acute Pain In the Emergency Setting
}

\author{
Özgür Karcioğlu* \\ Department of Emergency Medicine, University of Health Sciences, Turkey
}

Submission: February 06, 2018; Published: February 16, 2018

*Corresponding author: Özgür Karcioğlu, Department of Emergency Medicine, University of Health Sciences, Istanbul Education and Research Hospital, 34098, Fatih, Istanbul, Turkey, Email: okarcioglu@gmail.com

Abstract

Acutely painful injuries and illnesses are frequently under treated in the emergency department (ED), which indicates need for higher level of education and training about the management of acute pain. Assessment comes first in order to manage and alleviate the actual sensation of pain of the patient. Evaluation of pain should be taken as a part of the "standard of care" for EDs worldwide. The patients' perception of pain should be recorded upon the first encounter with the patient. The efficient management of pain in the acute setting requires proper evaluation as indicated via the patient's own statements, using a validated pain scale. The objective of this review is to highlight the current status of the assessment of pain with special emphasis on certeain techniques of evaluation, difficulties and opportunities thereof. Their use should inform our daily routine and pave the way to necessary improvements in the emergency evaluation and treatment of pain.

Keywords: Analgesia; Pain assessment; Pain management; Pain scale; Acute pain

\section{Introduction}

As a unique and subjective individual experience, acute pain is among the commonest chief complaints reported by a majority of patients who are admitted to the emergency department (ED), while its perception and expression have great variations between countries [1]. The International Association for the Study of Pain (IASP) has described it as 'an unpleasant sensory and emotional experience associated with actual or potential tissue damage, or described in terms of such damage' [2]. In the 17th century, poet and artist John Dryden (1631-1701) cited that "pain is the most common symptom reported to physicians; more than $80 \%$ of all patients who see physicians do so because of pain" [3]. Within the last two decades, pain management policies put forward by the Joint Commission on Accreditation of Healthcare Organizations (JCAHO) have become standard. In these guidelines, implementation of this standard in routine clinical practice comprised the acceptance of pain as the fifth vital sign recorded in the initial assessment; the use of pain intensity ratings; and posting of a statement on the management of pain in all settings.

The manifestation of pain in the acute conditionis related to the location, quality and severity of the negative feeling. Some authors supported the utilization of physiological variables to assess the quality of pain. Physiological clues such as tachycardia, hypertension or restlessness may help determine the requirement for pain management in intubated or agitated/incoherent patients [4]. Additional findings that suggest pain in the unconscious patient may be agitation, sweating, pallor, pupillary dilatation, moaning, grimacing and flinching [5]. Furthermore, abdominal and thoracic pain may result decline of tidal volume, vital capacity, and finally, alveolar hypoventilation [6]. Pain also stimulates intestinal secretions and increase smooth muscle sphincter tone. Boosted systemic vascular resistance and changes in sympathetic tone caused by pain candisguise volume depletion, thus may hinder adequate fluid resuscitation. In addition, persistent pain stimulates the pituitary-adrenal axis, which can inhibit the immune system and result in protracted wound healing and a propensity to postsurgical infection. Although there seems to be a consensus among medical disciplines to address its importance, one cannot yet claim that all healthcare personnel behave within standards in every real life situations worldwide.

\section{Perception and Assessment of Pain in the Acutely Ill}

Pain is what the patient tells it is. On the other hand, amyriad of psychological, sociocultural, time-dependent and conditional variables affects how people feel and describe their pain. Age, sex, ethnicity, associated psychiatric problems and socioeconomic status of the patient are some of the factors which 
affect the magnitude and quality of an individual expresses his/ her complaints. Subjective and multidimensional nature of the pain experience render evaluation of pain really challenging. The patient's perception and description of pain includes the sensory, emotional, psychological, and cultural components of the pain experience, therefore it cannot be defined via the unidimensional tools typically used in practice. Two decades ago, Raftery et al. [7] pointed out thatwomen reported the severity of pain more commonly than males did and therefore received more pain medications [7]. Thus the painful experience is a unique case for each individual, which warrants use of different tools of assessment in the routine practice.

There are at least 3 major foundations for prioritizing pain assessment:

a. Medical care quality,

b. Patient satisfaction, and

c. Compliance with regulatory requirements for assessing and documenting pain levels [8].

Thus, evaluation of pain is thought a part of the "standard of care" for EDs worldwide. In accord with this concept, the Agency for Health Care Policy and Research in the US pointed out that management of pain necessitatecooperation between all members of the health care team throughout the patient's course of treatment [9]. The association between pain severity and treatment requirements can be intervened by many factors inherent in the patient and the disease. Patients, families, nurses, and physicians feel better about pain care when pain levels are assessed [10-12]. Supplemented with regular pain reassessments, the schedule of pain reassessment should be driven by patients' pain severity [10].

\section{When should the patients' pain be queried?}

"Pain cannot be treated if it cannot be assessed". Pain should be assessed and documented within a short time after admission. Emergency healthcare personnel should address and record the patient's pain and discuss the plan for treatment whether or not pain is treated. The patients' perception of pain should be documented during the initial assessment of a patient. Current evidence provides a general recommendation that pain needs to be evaluated and managed within 20-25 minutes of initial healthcare provider assessment in the ED [13].

In a prospective, convenience-sample trial of all-diagnosis adults with pain at least $3 / 10$, Lozner et al. demonstrated that ED patients' preference for pain assessment is approximately 15 min, with more frequent intervals preferred when pain is severe [10]. Hatherley et al. [14] reviewed 15 researches to examine best practice related to acute pain assessment, documentation and management [14]. They postulated that mandating pain score reporting, pain assessment and reevaluation within predefined timeframes and analgesia within $30 \mathrm{~min}$ of admission would be most appropriate. They also pointed out that a higher incidence of pain assessment, reassessment and pain score documentation was well correlated with decreased time to analgesia. Nurse-led analgesia protocols are to be encouraged to increase incidence of documented pain assessment and shorten time to analgesia.

\section{Pain scores and documentation of pain}

Clinicians should evaluate and record their patients' pain levels, no matter which method or scale one uses to accomplish this task [15]. One definition of measurement is 'the assignment of numbers to objects or events to represent quantities of attributes according to rules' [16]. Children or patients with altered mental status are among these for whom pain assessmentcan represent a challenge [17]. Special scales developed and validated for patients with difficult communication are available, and ED physicians should have a plan for assessing pain in different case scenarios. Conscious patients are able to express and quantify their pain depending on personal characteristics, educational background, age, sex and other factors. The patient's self-report is the most accurate and reliable proof of the presence of pain and its intensity, and this holds true for patients of all ages, regardless of communication or cognitive deficits [18]. When the patient is capable of reporting pain, the patient's behavior or vital signs should never be used instead of self-report.

In the absence of objective measures, the clinician must depend on the patient to supply key information on the localization, quality and severity of the pain. Although physicians commonly question the reported severity and rely on their own estimates, the value of the patients' description of the location and nature of the discomfort has been proved on the theorethical basis and routine practice [19]. Interestingly, many researchers cited that physicians and nursesmostly underestimate and undertreat the patients' pain. Specific evidence undoubtedly indicates that physicians hardly predict how much pain their patients are feeling [20-23].

Scales devised to estimate and/or express the patient's pain can be evaluated in two groups: Unidimensional and multidimensional measures. It should be noted thatunidimensional scales measure only intensity, they cannot be viewed as a comprehensive pain assessment.Comprehensive pain assessment is expected to encompass both the unidimensionalmeasurement of pain intensity and the multidimensional evaluation of the pain perception.

The unidimensional pain intensity scales commonly used bedside are:

a. Numeric Rating Scale (NRS),

b. Visual Analog Scale (VAS),

c. Verbal Descriptor Scale (VDS).

The Visual Analog Scale (VAS): It is the most widely used tool for estimating both severity of pain and to judge the extent of pain relief [24-26]. Healthcare worker asks the patient to select a point on a line drawn between two ends to express how intense he/she perceives pain (Figure 1). The VAS is an easy-to-use 
instrument which does not warrant using a sophisticated device. It is also highly sensitive in detecting treatment effects, and its results can be analysed by parametric tests $[25,27]$. Although this tool is suitable for use with older children and adults, the need for a marking and for being able to visualize and mark the line, can make the VAS impractical to use in the emergency situation.

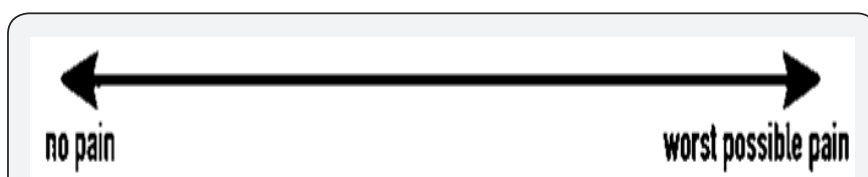

Figure 1: A visual analog scale (VAS).

However, there is a tendency to overestimate the clinical importance of a small difference in VAS score. Since the recorded statistical differences might not represent clinically important changes in pain or truly meaningful differences to patients, considerable emphasis has been put on measuring the "minimum clinically significant difference" (MCSD) in acute analgesia research [24,25,28-30]. The term MCSD is used to describe the change in pain necessary to be perceived clinically by the patient and was measured as $13 \mathrm{~mm}$ on a $100 \mathrm{~mm}$ VAS, while physicianperceived change was measured as high as $18 \mathrm{~mm}$ [25]. This phenomenon may suggest physicians are some what likely to underestimate the changes of pain in their patients. The MCSD of ED patients with nontraumatic thoracoabominal pain in Turkey is $24.2 \mathrm{~mm}$ and it is not affected by gender, age, level of education, location, or duration of pain [31].

The numeric rating scale (NRS): It is broadly validated instrument across a wide array of patient types. Data obtained via NRS are easily documented, intuitively interpretable, and meet regulatory requirements for pain assessment and documentation [32]. To date, findings demonstrated that even in the chaotic prehospital phase most acute care patients allow evaluation via a simple "zero-to-10 scale" or NRS reliably, respecting their pain levels [33]. Evidence indicated that patients reallywant to give a pain number, rather than simply relate whether they want analgesia.

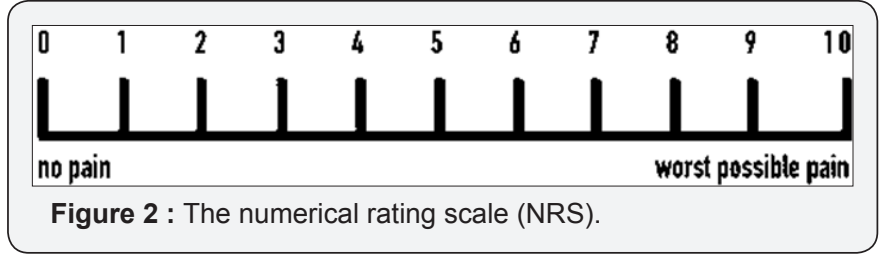

NRS is a commonly used tool necessitating the patient rate his pain on a scale from 0 to 10 , with 0 indicating no pain and 10 reflecting the worst possible pain (Figure 2). NRS are often conducted as a scale from 1 to 10 which does not give the patient a solution to indicate no pain at all. It can be used with children who are able to understand numbers. The pain scores are interpreted as:
a. $0=$ no pain
b. 1-3 = mild pain

c. $\quad 4-6=$ moderate pain

d. $\quad 7-10=$ severe pain

NRS can be used with most children older than 8 years of age, and behavioral observation scales are required for those unable to provide a self-report [34]. For patients with cancer-related pain, the NRS is the most frequently used instrument to measure pain intensity [35].

Verbal pain scores (VPSs), Verbal rating scales (VRS) or Verbal Descriptor Scales: These tools may discern those patients who suffer from pain but who may not express their discomfort, as well as influence the physician to inquire about the patient's pain. A prospective study introducing VPSs in an ED revealed that of those trauma patients who had VPS scores documented, $60 \%$ received analgesics versus $33 \%$ in those who did not have a VPS score documented [36]. Furthermore, those with higher VPS scores were more likely to receive analgesics. Todd et al. [37] disclosed that in patients who were not treated with analgesics, $42 \%$ desired them, but only one-third of the sample voiced their concern [37].

\begin{tabular}{|ccc|}
\hline None Mild & Moderate & Severe \\
Choose the word that best describes your pain. & \\
\hline
\end{tabular}

Figure 3 : Verbal rating scale.

Pines et al. [38] reported that ED crowding has been demonstrated to boost time to analgesic administration and death rate. The use of VPS in this setting may identify those individuals in need of more expedient treatment.Verbal rating scales (VRS) or Verbal Descriptor Scales consist of a number of statements describing increasing pain intensities (Figure 3). Patients are told to choose the word which best describes their pain intensity. The number of descriptors used has ranged from four (none, mild, moderate, severe) to 15 [39]. For patients who have limited literacy or cognitive impairment, use of these scales may be difficult, and they do not provide the number of choices available with the VAS or NRS, thus potentially limiting precision [40].

McLean et al. [33] conducted a retrospective cross-sectional study of emergency medical services (EMS) run sheets after the adoption of a universal prehospital pain assessment protocol [33]. Prehospital pain assessment using a VRS and NRS was feasible in this patient population. The greatest risk factor for being unable to assess and record pain levels was altered mental status (39\% of those not assessed). Among patients reporting pain, one-fifth completed a VRS but not a NRS.

Some investigators tried to compare pain scales used in the acute care setting and to find out which performed best in the management of the patients. In a recent study Bahreini et al. [41] compared three self-report pain scales in adults with acute pain [41]. VAS, Color Analog Scale (CAS), and verbally administered NRS were found to have strong correlation between each other. The authors concluded that the three scales can be interchangeably 
applied for acute pain assessment in adult patients. Likewise, Hjermstad et al. [42] performed a comparison study of unidimensional pain scales as part of the work of the European Palliative Care Research Collaborative on pain assessment [42]. They reported that NRSs had better compliance in 15 of 19 studies reporting pain intensity, when compared to the VAS and VRS, and thus were the instrumentof choice in 11 studies on the basis of higher compliance rates, better responsiveness and ease of use, and better applicability relative to VAS and/or VRS. On the other hand, 29 studies yielded no preference.

\section{Non-Verbal Scale}

Faces Pain Scale - Revised (FPS-R): It is a self-report measure of pain intensity developed for young children [43]. It was adapted from the FPS to enable scoring and recording the severity of pain on the widely accepted 0 -to-10 metric (Figure 4) [44]. The scale consists of six faces showing progressive pain intensities, beginning with a smiling face and ending with a crying face. The scale shows a close linear relationship with VAS between the ages of 4 and 16 years. Main advantages include ease of application and lack of additional requirements forusage except for the photocopied faces and a marker to accomplish the task in the busy ED environment.

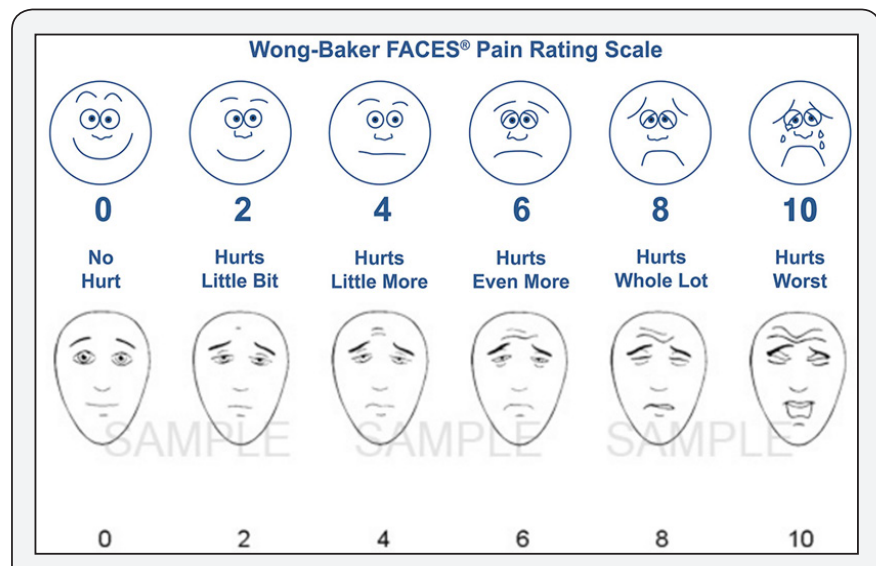

Figure 4 : FACES Pain rating scale.

Moutte et al. [45] conducted a study using a prestructured questionnaire on 75 emergency physicians in Norway [45]. They reported that a pain scale with a VAS had been used by $59 \%$ of physicians in patients between 9 to 19 years of age, by $23 \%$ in children aged 3 to 8 years, and by $3 \%$ in children below 3 years. Emergency physicians report a need for pain assessment procedures in children and youth. Olsen et al. [46] conducted a study to measure the impact of implementing apain management algorithm in adult intensive care unit (ICU) patients expressing their pain. The number of pain assessments was higher in the intervention group compared with the control group. Interestingly, duration of ventilation and length of ICU stay decreased significantly in the intervention group compared with the control group. Several outcome variables were significantly improved after implementation of the algorithm compared with the control group. Instruments for behavioral assessment are helpful in detecting the magnitude of pain and evaluating approaches to treatment.

These scales are of two types:

a. Pain behavior scales, and

b. Pain behavior checklists.

Some of these scales are scored by identifying the number or intensity of behaviors. However, no research as yet confirms that a pain behavior score is a pain intensity score [47]. Therefore, it is unsafe to use pain behavior scores as pain intensity scores. A patient with only a few behaviors may have as much pain as a patient with many more behaviors. An example of a pain behavior scale is the Behavioral Pain Scale (BPS), developed for use in the critically ill patient in the ICU [48]. It evaluates and scores three categories of behavior:

a. Facial expression, scores range from 1 for relaxed to 4 for grimacing

b. Upper-limb movement, scores range from 1 for no movement to 4 for permanently retracted

c. Ventilator compliance, scores range from 1 for tolerating ventilator to 4 for unable to control ventilation

A score above 3 may indicate pain is present and the score can be used to evaluate intervention, but cannot be interpreted to mean pain intensity. For a pain behavior scale to be useful, the patient must be able to respond in all categories of behavior. For example, the BPS would be useless in a patient who is receiving a neuromuscular blocking agent. Brief Pain Inventory (BPI) is a selfadministered, validated questionnaire available in many languages and was originally used to assess pain intensity and location as well as pain-related functional interference among cancer patients (Figure 5) [49]. It was also translated and validated in different cultures and disease settings. In a recent study conducted on Thai cardiac patients, Keawnantawat et al. [50] reported that the BPI-T also has good internal consistency (Cronbach's alpha coefficients 0.76 and 0.85 ) [50]. Pearson's correlation coefficients at 0.35 to 0.70 supported the convergent validity to the NRS.

The functional interference section of the BPI assesses the impact of pain on various aspects of daily functioning. Using a 0 to 10 scale $(0=$ does not interfere, $10=$ completely interferes), the patient is asked to rate the degree to which pain interferes with seven functions during the past 24 hours: general activity, mood, walking ability, normal work, relations with other people, sleep, and enjoyment of life [51]. Investigators have modified the original BPI interference scale to include additional domains such as recreational and social activities as well as self-care [52,53]. Lapane et al. [54] compared two-factor representation (pain intensity and interference) and three-factor representation (pain intensity, activity interference, and affective interference) of the modified BPI and reported that a two-factor representation of BPI is appropriate among cancer patients [54]. In an analysis involving 
data from four countries, the scale displayed internal stability coefficients from 0.86 to 0.91 [55]. Test-retest reliability at 30 minutes was found to be excellent in one German study(r=0.97) [56].

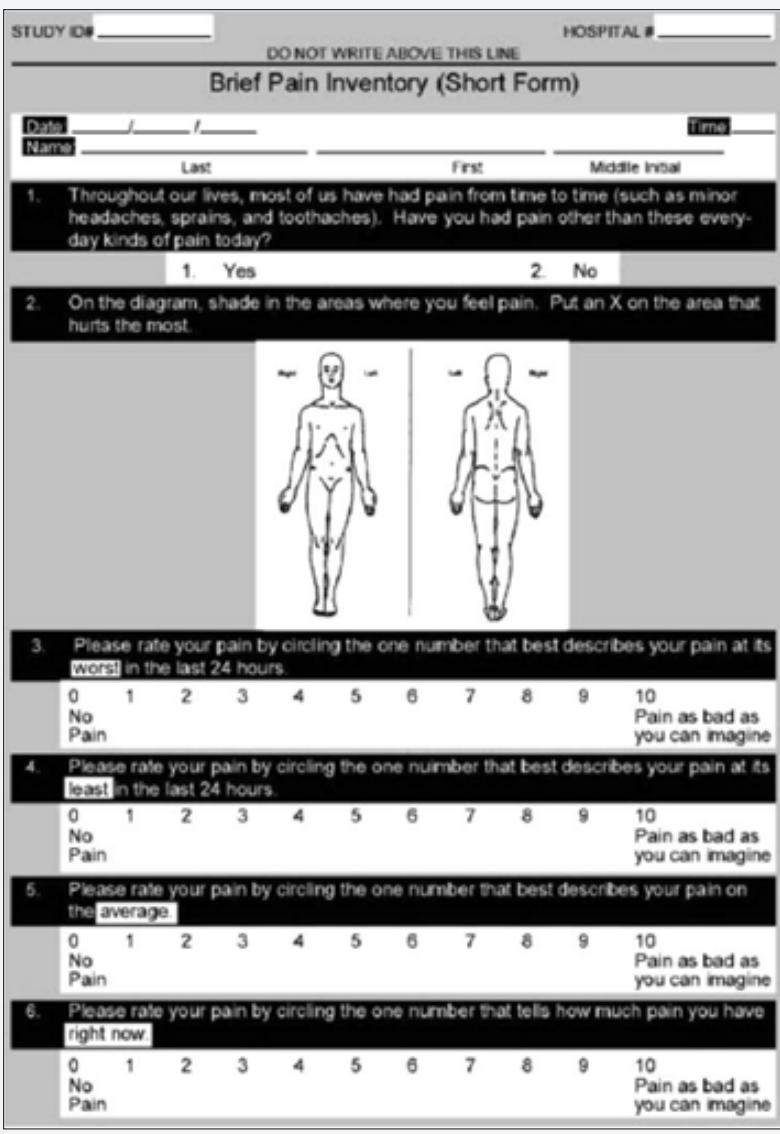

Figure 5 : The Brief Pain Inventory.

Cross-cultural and sociodemographic differences in pain assessment: Dale et al. [57] put forward that women had a higher probability of not being inquired about pain, but there was no difference in the ratio of patients asked about pain with respect to age. Additionally, patients with hypoxia and hypotension were less likely to be cared about their pain. They concluded that evaluation and treatment of pain in the ED are insufficient and not fully compatible with the local protocols. A focus on strategies to improve pain treatment in the ED is a necessary aspect of developing sufficient care.

A study on cancer-related painfrom Netherlands reported that measurement of "acceptable pain intensity" is feasible and almost half of the patients scored their acceptable pain in the range of moderate to severe [35]. Recent reports launch considerable aid derived from technological advances in the assessment and recording of pain. Kos et al. [58] conducted a cross-sectional observational study using smartphones and tablets to score statements assessing fatigue, pain, anxiety and quality of life on an electronic visual analogue scale (eVAS) [58]. They concluded that eVAS are reliable and useful for people with multiple sclerosis to register their complaints. Arthur et al. [12] conducted a pilot

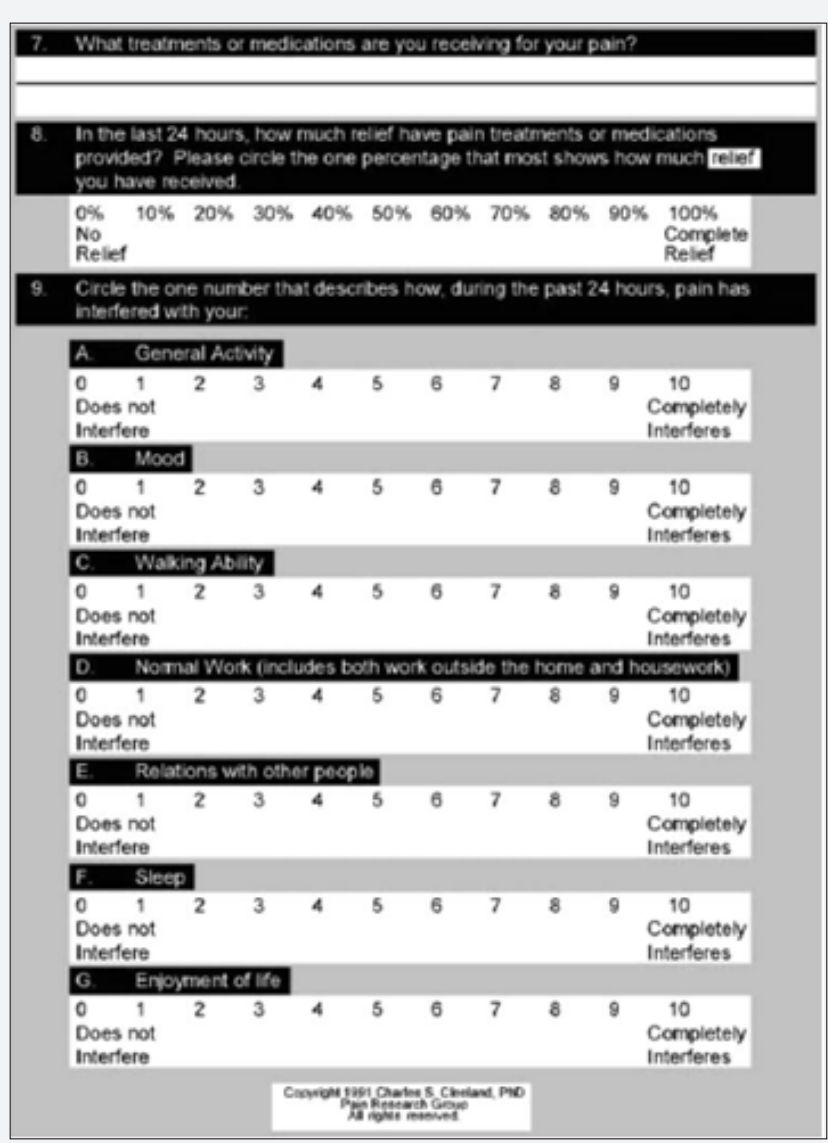

project to reveal the feasibility of the tablet computer-based automated pain tracker (APT) and to assess patient and nurse satisfaction with the device. All of the subjects indicated the APT was easy to use, and 28 of 30 subjects (93\%) thought the APT should be used more frequently in the ED. They concluded that the iPad pain-tracking and reporting-system provided higher patient satisfaction, improved pain care, operational efficiency, and better documentation of pain evaluation and management.

Finally, a very recent systematic review analysed the VAS, the VRS and the NRS to determine the compliance and usability different among scales. The authors pointed out that all three scales are valid, reliable and appropriate for use in clinical practice, although the VAS faces more difficulties than the others. For general purposes the NRS has good sensitivity and generates data that can be analysed for audit purposes [59].

\section{Conclusion}

"Pain cannot be treated if it cannot be assessed". The most important principle is that clinicians should some how assess their patients' pain levels, no matter which method or scale one uses to accomplish this task. Special scales developed and validated 
for patients with difficult communication are available, and ED physicians should have a plan for assessing pain in different case scenarios. Finally, ongoing research in the area of acute pain management should be conducted.

\section{References}

1. Li SF, Greenwald PW, Gennis P, Bijur PE, Gallagher EJ (2001) Effect of age on acute pain perception of astandardized stimulus in the emergency department. Ann Emerg Med 38(6): 644-647.

2. (1994) Part III: Pain Terms, A Current List with Definitions and Notes on Usage. In: H Merskey \& N Bogduk (Eds.), Classification of Chronic Pain, $\left(2^{\text {nd }} e d n\right)$, IASP Task Force on Taxonomy, IASP Press, Seattle, USA, pp. 209-214.

3. (2017) Bajwa ZH, Wootton RJ, Warfield CA (Eds). Principles and Practice of Pain Medicine. ( $3^{\text {rd }}$ edn), McGraw-Hill Education, China.

4. Murray MJ (1990) Pain problems in the ICU. Crit Care Clin 6(2): 235253.

5. Kaiser KS (1992) Assessment and management of pain in the critically ill trauma patient. Crit Care Nurs Q 15(2): 14-34.

6. Ossewaarde SB (1997) Pain Management Practice in the Acute Adult Trauma Patient. Masters Theses.333 p. 1-50.

7. Raftery KA, Smith Coggins R, Chen AH (1195) Gender-associated differences in emergency department pain management. Ann Emerg Med 26(4): 414-421.

8. Ducharme J (2008) Introduction and general approach to pain. In: Thomas SH (Ed.), Emergency Department Analgesia: An Evidence Based Guide, Cambridge University Press, Cambridge, UK, p. 3-9.

9. Acute pain management: operative or medical procedures and trauma, Part 2. Agency for Health Care Policy and Research. Clin Pharm 11(5): 391-414.

10. Lozner AW, Reisner A, Shear ML, Patel S, Connolly J, et al. (2010) Pain severity is the key to emergency department patients' preferred frequency of pain assessment. Eur J Emerg Med 17(1): 30-32.

11. Thomas SH, Andruszkiewicz LM (2004) Ongoing visual analog score display improves Emergency Department pain care. J Emerg Med 26(4): 389-394.

12. Arthur AO, Whiteside S, Brown L, Minor C, Thomas SH (2012) Patient Use of Tablet Computers to Facilitate Emergency Department Pain Assessment and Documentation. ISRN Emerg Med 2012(2012): 6.

13. Grant PS (2006) Analgesia delivery in the ED. Am J Emerg Med 24(7): 806-809.

14. Hatherley C, Jennings N, Cross R (2016) Time to analgesia and pain score documentation best practice standards for the Emergency Department - A literature review. Australas Emerg Nurs J 19(1): 26-36.

15. Thomas SH (2013) Management of Pain in the Emergency Department. ISRN Emerg Med 2013(2013): 19.

16. Chapman CR (1976) Measurement of pain: problems and issues. In: Bonica JJ \& Albe-Fessard D (Eds.), Advances in pain research and therapy, Raven Press, New York, USA, 1: 345-353.

17. Voepel Lewis T, Merkel S, Tait AR, Trzcinka A, Malviya S (2002) The reliability and validity of the face, legs, activity, cry, consolability observational tool as a measure of pain in children with cognitive impairment. Anesthesia\& Analgesia 95(5): 1224-1229.

18. (1992) Acute pain management: Operative or medical procedures and trauma. Agency for Health Care Policy and Research, Acute Pain Management Guideline Panel. Clinical practice guidelines. U.S. Department of Health and Human Services, Rockville, MD, USA.
19. Perry S, Heidrich G (1982) Management of pain during debridement: A survey of US burn units. Pain 13(3): 276-280.

20. GouletJL, BrandtC, Crystal S, Fiellin DA, GibertC, et al. (2013) Agreement between electronic medical record-based and self-administered pain numeric rating scale: clinical and research implications.Med Care 51(3): 245-250.

21. Montali L, Monica C, Riva P, Cipriani R (2011) Conflictingrepresentations of pain: a qualitative analysis of health care professionals' discourse. Pain Med 12(11): 1585-1593.

22. Laugsand EA, Sprangers MAG, Bjordal K, Skorpen F, Kaasa S, et al. (2010) Health care providers underestimate symptom intensities of cancer patients: a multicenter European study. Health Qual Life Outcomes 8: 104.

23. Shugarman LR, Goebel JR, Lanto A, Asch SM, Sherbourne CD, et al. (2010) Nursing staff, patient, and environmental factors associated with accurate pain assessment. J Pain Symptom Manag 40(5): 723-733.

24. Kelly AM (2001) The minimum clinically significantdifference in visual analogue scale pain score does notdiffer with severity of pain. Emerg Med J 18(3): 205-207.

25. Gallagher EJ, Liebman M, Bijur PE (2001) Prospectivevalidation of clinically important changes in painseverity measured on a visual analog scale. Ann EmergMed 38(6): 633-638.

26. Huskisson EC (1974) Measurement of pain. Lancet 304(7889): 1127 1131.

27. Dexter F, Chestnut DH (1995) Analysis of the statistical teststo compare visual analog scale measurements among groups. Anesthesiology 82(4): 896-902.

28. Bird SB, Dickson EW (2001) Clinically significant changes in pain along the visual analog scale. Ann Emerg Med 38(6): 639-643.

29. Todd KH, Funk KG, Funk JP, Bonacci R (1996) Clinicalsignificance of reported changes in pain severity. AnnEmerg Med 27(4): 485-489.

30. Gallagher EJ, Bijur PE, Latimer C, Silver W (2002) Reliability and validity of a visual analog scale for acute abdominal pain in the emergency department. Am J Emerg Med 20(4): 287-290.

31. Degerli V, Karcioglu O, Larkin GL, Karaduman S, Korkmaz T, et al. (2012) The minimum clinically significant difference in acute nontraumatic thoracoabdominal pain in adult emergency department patients in Turkey. Hong Kong J Emerg Med 19(3): 171-176.

32. Marco CA, Marco AP (2008) Assessment of pain. In: Emergency Department Analgesia: An Evidence Based Guide, Thomas SH, (Eds.), Cambridge University Press, Cambridge, UK, p. 10-18.

33. McLean SA, Domeier RM, DeVore HK, Hill EM, Maio RF, et al. (2004) The feasibility of pain assessment in the prehospital setting. Prehosp Emerg Care 8(2): 155-1561.

34. Von Baeyer CL (2006) Children's self-reports of pain intensity: Scale selection, limitations and interpretation. Pain Res Manag 11(3): 157162.

35. Oldenmenger WH, van der Rijt CC (2017) Feasibility of assessing patients' acceptable pain in a randomized controlled trial on a patient pain education program. Palliat Med 31(6): 553-558.

36. Silka PA, Mendel MR, Moreno G, Merrill L, Geiderman JM (2008) Pain scores improve analgesic administration patterns for trauma patients in the emergency department. Acad Emerg Med 11(3): 264-270.

37. Todd K, Ducharme J, Choiniere M, Group PS (2007) Pain in the emergency department: results of the pain and emergency medicine (PEMI) multicenter study. J Pain 8(6): 460-466.

38. Pines JM, Hollander JE (2007) Emergency department crowding is associated with poor care for patients with severe pain. Ann Emerg Med 51(1): 1-5. 
39. Gracely RH, McGrath P, Dubner R (1978) Ratio scales of sensory and affective verbal pain descriptors. Pain 5(1): 5-18.

40. Ferraz MB, Quaresma MR, Aquino LR, Atra E, Tugwell P, et al. (1990) Raliability of pain scales in the assessment of literate and illiterate patients with rheumatoid arthritis. J Rheumatol 17(8): 1022-1024.

41. Bahreini M, Jalili M, Moradi Lakeh M (2015) A comparison of three selfreport pain scales in adults with acute pain. J Emerg Med 48(1): 10-18.

42. Hjermstad MJ, Fayers PM, Haugen DF, Caraceni A, Hanks GW, et al. (2011) European Palliative Care Research Collaborative (EPCRC) Studies comparing Numerical Rating Scales, Verbal Rating Scales, and Visual Analogue Scales for assessment of pain intensity in adults: a systematic literature review. J Pain Symptom Manage 41(6): 10731093.

43. Hicks CL, von Baeyer CL, Spafford P, van Korlaar I, Goodenough B (2001) The Faces Pain Scale - Revised: Toward a common metric in pediatric pain measurement. Pain 93(2): 173-183.

44. Bieri D, Reeve R, Champion GD, Addicoat L, Ziegler J (1990) The Faces Pain Scale for the self-assessment of the severity of pain experienced by children: Development, initial validation and preliminary investigation for ratio scale properties. Pain 41(2): 139-150.

45. Moutte SD, Brudvik C, Morken T (2015) Physicians' use of pain scale and treatment procedures among children and youth inemergency primary care - a cross sectional study. BMC Emerg Med 15: 33.

46. Olsen BF, Rustøen T, Sandvik L, Jacobsen M, Valeberg BT (2016) Results of implementing a pain management algorithm in intensive care unit patients: The impact on pain assessment, length of stay, and duration of ventilation. J Crit Care 36: 207-211.

47. Pasero C, McCaffery M (2005) No self-report means no pain-intensity rating. Am J Nurs 105(10): 50-53.

48. Payen JF, Bru O, Bosson JL, Lagrasta A, Novel E, et al. (2001) Assessing pain in critically ill sedated patients by using a behavioral pain scale. Crit Care Med 29(12): 2258-2263.

49. Cleeland CS, Ryan KM (1994) Pain assessment: Global use of the Brief Pain Inventory. Ann Acad Med 23(2): 129-138.
50. Keawnantawat P, Thanasilp S, Preechawong S (2017) Translation and Validation of the Thai Version of a Modified Brief Pain Inventory: A Concise Instrument for Pain Assessment in Postoperative Cardiac Surgery. Pain Pract 17(6): 763-773.

51. (2009) Cleeland CS (Ed.), The Brief Pain Inventory User Guide.

52. Tyler EJ, Jensen MP, Engel JM, Schwartz L (2002) The reliability and validity of pain interference measures in persons with cerebral palsy. Arch Phys Med Rehabil 83(2): 236-239.

53. Jensen MP, Ehde DM, Hoffman AJ, Patterson DR, Czerniecki JM, et al. (2002) Cognitions, coping and social environment predict adjustment to phantom limb pain. Pain 95(1-2): 133-142.

54. Lapane KL, Quilliam BJ, Benson C, Chow W, Kim M (2014) One, two, or three? Constructs of the brief pain inventory among patients with noncancer pain in the outpatient setting. J Pain Symptom Manage 47(2): 325-333.

55. Serlin RC, Mendoza TR, Nakamura Y, Edwards KR, Cleeland CS (1995) When is cancer pain mild, moderate or severe? Grading pain severity by its interference with function. Pain 61(2): 277-284.

56. Radbruch L, Loick G, Kiencke P, Lindena G, Sabatowski R, et al. (1999) Validation of the German version of the Brief Pain Inventory. J Pain Symptom Manage 18(3): 180-187.

57. Dale J, Bjørnsen LP (2015) Assessment of pain in a Norwegian Emergency Department. Scand J Trauma Resusc Emerg Med 23: 86.

58. Kos D, Raeymaekers J, Van Remoortel A, Dhooghe, Nagels G, et al. (2017) Electronic visual analogue scales for pain, fatigue, anxiety and quality of life in people with multiple sclerosis using smartphone and tablet: a reliability and feasibility study. Clin Rehabil 31(9): 1215-1225.

59. Karcioglu O, Topacoglu H, Dikme O, Dikme O (2018) A systematic review of the pain scales in adults: Which to use? Am J Emerg Med pii: S0735-6757(18)30008-1.

\section{Your next submission with Juniper Publishers} will reach you the below assets

- Quality Editorial service

- Swift Peer Review

- Reprints availability

- E-prints Service

- Manuscript Podcast for convenient understanding

- Global attainment for your research

- Manuscript accessibility in different formats

( Pdf, E-pub, Full Text, Audio)

- Unceasing customer service

Track the below URL for one-step submission https://juniperpublishers.com/online-submission.php 